Anaesthesist 2010 - 59:1083-1090 DOI 10.1007/s00101-010-1795-6

Eingegangen: 20. Januar 2010

Überarbeitet: 15. Juli 2010

Angenommen: 19. August 2010

Online publiziert: 12. November 2010

Redaktion

R. Rossaint, Aachen

C. Lichtenstern ${ }^{1}$ J. Pratschke ${ }^{2} \cdot$ U. Schulz ${ }^{3} \cdot$ M. Schmoeckel ${ }^{4} \cdot$ W. Knitsch ${ }^{5}$.

P. Kaskel ${ }^{6} \cdot$ K.J. Krobot ${ }^{6} \cdot$ M.A. Weigand ${ }^{1,7} \cdot$ M. Winkler ${ }^{5}$

${ }^{1}$ Klinik für Anästhesiologie und Operative Intensivmedizin,

Universitätsklinikum Gießen und Marburg, Standort Gießen

${ }^{2}$ Klinik für Viszeral- und Transplantationsmedizin, Charité - Universitätsmedizin Berlin

${ }^{3}$ Klinik für Thorax- und Kardiovaskularchirurgie, Herzzentrum Nordrhein-

Westfalen, Ruhr-Universität Bochum, Bad Oeynhausen

${ }^{4}$ Herzchirurgische Klinik, Klinikum der Ludwig-Maximilians-Universität, München-Großhadern

${ }^{5}$ Klinik für Viszeral- und Transplantationschirurgie, Medizinische Hochschule Hannover

${ }^{6}$ Outcomes Research, MSD SHARP \& DOHME GmbH, Haar

${ }^{7}$ Klinik für Anästhesiologie, Universitätsklinikum Heidelberg

\title{
Caspofungin nach Transplantation solider Organe in Deutschland
}

\author{
Beobachtungsstudie zur Behandlung \\ invasiver Pilzinfektion
}

temische Pilzinfektionen gehören zu den klassischen opportunistischen Erkrankungen bei transplantierten Personen, mit hohen Morbiditäts- und Letalitätsraten $[23,24,26]$. Die Inzidenz von Pilzinfektionen variiert je nach Quelle und transplantiertem Organ deutlich [16]. So liegt die Rate der systemischen Pilzinfektionen nach Lebertransplantationen mit 4-50\% am höchsten, während sie für thorakale Transplantationen mit $15-35 \%$ und für Nierentransplantationen mit $2-14 \%$ angegeben wird [9]. Diese Pilzinfektionen werden vorwiegend durch Candida- und Aspergillus-Spezies verursacht [25]. Die zumeist chronisch bestehende Organinsuffizienz und weitere Erkrankungen (Z. B. Diabetes, chronische Niereninsuffizienz, Kachexie) stellen wichtige prädisponierende Faktoren dar, die mit einer Immunschwäche einhergehen. Im Zusammenhang mit systemischen Mykosen wurden für Transplantationspatienten als Risikofaktoren eine präoperative Kolonisation mit Candida, ein pulmonaler Asper-
gillus-Nachweis (Lungentransplantation), eine Retransplantation, eine lange Operationsdauer $(>5 \mathrm{~h})$ mit hohem Blutverlust/ Massivtransfusion $[19,28]$, ein langer Intensivaufenthalt zusammen mit der Notwendigkeit der Anlage invasiver Katheter (Z. B. zentraler Venen-, Dialysekatheter, Drainagen, Stents; $[10,18,20,28])$, der Einsatz von Breitspektrumantibiotika, ein Nierenversagen (ggf. Hämofiltration bzw. Dialyse; $[7,10])$, eine akute bzw. chronische Transplantatdysfunktion und eine parallele virale Infektion [insbesondere Zytomegalievirus (CMV) [6, 7]] identifiziert. Aufgrund der besonderen Beeinflussung der T-Zell-vermittelten spezifischen Immunität durch Immunsuppressiva sowie durch die parallel verabreichten Kortikoide und deren hemmenden Einflüsse auf die angeborene Immunität ist regelmäßig an die Entwicklung einer Pilzinfektion zu denken. Neben dem er-

(c) The authors 2010. This article is published with open access at Springerlink.com. deutsames Risiko für Patienten nach Transplantation solider Organe dar. Sys- 
Tab. 1 Listung der im Rahmen von CAPSOT retrospektiv erhobenen Grunderkrankungen und Risikofaktoren für invasive Pilzerkrankungen

\begin{tabular}{|c|c|}
\hline Grunderkrankungen & Risikofaktoren für invasive Pilzerkrankungen \\
\hline Angeborene Erkrankungen & Akute maligne Erkrankung \\
\hline Endokrine Erkrankungen & Dialysepflichtiges Nierenversagen \\
\hline Erkrankungen des Gastrointestinaltrakts & Primäres Tranplantatversagen \\
\hline Genitourinale Erkrankungen & Retransplantation \\
\hline Herzerkrankungen & Reexploration \\
\hline Hämatologische Erkrankungen & Therapie mit mehr als 3 Antibiotika \\
\hline Bluthochdruck & Kürzlich erfolgte Anlage eines zentralen Venenkatheters \\
\hline Erkrankungen der Leber & $\begin{array}{l}\text { Steroidgabe während des aktuellen Krankenhaus- } \\
\text { aufenthalts }\end{array}$ \\
\hline Erkrankungen der Lungen & Hyperglykämie \\
\hline Neoplastische Erkrankungen & Neutropenie (ANC <500 Zellen/ $\mu$ l) \\
\hline $\begin{array}{l}\text { Neurologisch-/psychiatrische } \\
\text { Erkrankungen }\end{array}$ & $\begin{array}{l}\text { Kürzlich erfolgte parenterale Ernährung } \\
\text { (Hyperalimentation) }\end{array}$ \\
\hline $\begin{array}{l}\text { Erkrankungen des rheumatischen For- } \\
\text { menkreises und des Bewegungsapparats }\end{array}$ & Klasse 1 des United Network for Organ Sharing \\
\hline Hauterkrankungen & Notoperation \\
\hline Gefäßerkrankungen & $\begin{array}{l}\text { Nachweis einer Kolonisation durch Pilze vor Organ- } \\
\text { transplantation }\end{array}$ \\
\hline \multirow[t]{10}{*}{ Sonstige } & Zytomegalievirusinfektion \\
\hline & Lange Dauer der Organtransplantation ( $>5 \mathrm{~h}$ ) \\
\hline & Biliäre Junostomie mit Roux-Y-Anastomose \\
\hline & $\begin{array}{l}\text { Reoperation (Laparotomie) innerhalb von } 5 \text { Tagen nach } \\
\text { Organtransplantation }\end{array}$ \\
\hline & $\begin{array}{l}\text { Erhöhte Transfusionsgabe während der Organtrans- } \\
\text { plantation ( } \geq 40 \text { Einheiten) }\end{array}$ \\
\hline & Überlastung der Leber mit Eisen \\
\hline & Thrombozytopenie \\
\hline & Fulminantes Leberversagen \\
\hline & $\begin{array}{l}\text { Kürzlich erfolgter Aufenthalt auf einer Intensivstation } \\
\text { während des Beobachtungszeitraums }\end{array}$ \\
\hline & $\begin{array}{l}\text { Umgebungs-/ bzw. in der Öffentlichkeit stattgefundene } \\
\text { Exposition invasiver Pilzerreger }\end{array}$ \\
\hline
\end{tabular}

weiterten Erregerspektrum unterscheiden sich auch die klinischen Symptome und der Verlauf der Infektionserkrankungen, die unter Immunsuppression symptomärmer und beschleunigt ablaufen können.

\section{Vorab geplante Sekundäranalyse der deutschen Daten einer multi- nationalen Beobachtungsstudie}

\section{Patienten und Methoden}

Diese Studie ist Teil einer retrospektiven internationalen multizentrischen Beobachtungsstudie, die transplantierte Patienten aus Deutschland, Großbritannien, Italien und China untersuchte [29].
Die Untersuchung wurde den zuständigen Ethikkommissionen entsprechend den Regularien vor Ort vorgelegt. Die hier vorgestellte länderspezifische Sekundäranalyse der in Deutschland teilnehmenden Patienten war gemäß Studienprotokoll und statistischem Analyseplan vorab geplant.

Konsekutiv wurden organtransplantierte Patienten in die Studie aufgenommen, die im Zeitraum von Januar 2004 bis Januar 2007 im Zusammenhang mit einer Transplantation an einem der Studienzentren wegen einer gesicherten oder wahrscheinlichen invasiven Pilzinfektion behandelt worden waren. Hierbei wurden von dem behandelnden Arzt die Kriterien der European Organization for Research and Treatment of Cancer/Mycoses Study Group (EORTC-MSG) als Leitlinie für die Einschätzung verwendet, ob die invasive Pilzinfektion wahrscheinlich oder bewiesen war [1]. Die Entscheidung zur Durchführung diagnostischer Maßnahmen bzw. für die Behandlung mit Caspofungin hatte der behandelnde Arzt auf Basis der individuellen klinischen Beurteilung sowie lokalen Diagnose- und Behandlungsstandards und unabhängig von der aktuellen Erhebung getroffen. Caspofungin konnte dabei entweder zur primären oder sekundären Therapie sowie als Mono- oder Kombinationspräparat verabreicht worden sein. Im Rahmen dieser retrospektiven Beobachtungsstudie wurde keine Studienmedikation gestellt; die Dosierung wurde vom individuellen Arzt auf Basis der Fachinformation [17] und lokalen Behandlungsstandards für den jeweiligen Patienten festgelegt. Die retrospektive Durchsicht der Krankenakten erfolgte zwischen Juli 2007 und Dezember 2007. Im Fall einer Retransplantation wurde die Transplantation mit kürzestem Abstand zur Pilzinfektion berücksichtigt. Die Patienten hatten während des Krankenhausaufenthalts nicht an anderen klinischen Studien zur Therapie der invasiven Pilzerkrankung teilgenommen. Erhoben wurden:

- demografische Merkmale des Teilnehmers,

- Gesundheitszustand zum Zeitpunkt der Transplantation, einschließlich Art und Dauer der Organtransplantation („solid organ transplantation“, SOT) sowie der Grund für die SOT,

- Grunderkrankungen (• Tab. 1),

- Risikofaktoren für invasive Pilzerkrankungen (• Tab. 1),

- Daten zum Krankenhausaufenthalt,

- Daten zur antimykotischen Prophylaxe und Therapie, einschließlich immunmodulatorischer Therapie und Gesundheitszustand des Patienten zu Beginn, während und zum Ende der antimykotischen Therapie,

- mikrobiologische Befunde einschließlich Art und Lokalisation der Pilzerkrankung sowie die im Rahmen der Diagnose und Behandlung durchgeführten diagnostischen Maßnahmen, 


\section{Lichtenstern · J. Pratschke · U. Schulz · M. Schmoeckel · W. Knitsch · P. Kaskel · K.J. Krobot · M.A. Weigand · M. Winkler Caspofungin nach Transplantation solider Organe in Deutschland. Beobachtungsstudie zur Behandlung invasiver Pilzinfektion}

\section{Zusammenfassung}

Hintergrund. Diese Studie ist eine vorab geplante Sekundäranalyse der deutschen Daten einer multinationalen multizentrischen Beobachtungsstudie. Ziel dieser retrospektiven Studie war es, die Alltagswirksamkeit („,effectiveness") von Caspofungin bei organtransplantierten Patienten mit wahrscheinlicher oder nachgewiesener invasiver Pilzerkrankung zu evaluieren.

Material und Methoden. Die Daten wurden bei Patienten, die von Januar 2004 bis Juni 2007 organtransplantiert worden waren, zu jeweils einer Episode einer invasiven Pilzinfektion retrospektiv erhoben. Die Daten zur Alltagswirksamkeit wurden für Patienten berichtet, die mit mindestens 5 Dosen Caspofungin behandelt worden waren. Die Daten zur Sicherheit wurden für alle Patienten ausgewertet, die mindestens eine Dosis Caspofungin erhalten hatten. Sämtliche Auswertungen erfolgten deskriptiv.

Ergebnisse. Es wurden 41 organtransplantierte Patienten [davon 14 Frauen; Alter (Median) 56 Jahre, Acute Physiology and Chronic Health Evaluation (APACHE) II Score zu Beginn der Caspofungintherapie (Median) 23] aus 5 deutschen Zentren in die Studie aufgenommen, v. a. Herz- (51\%) und Lebertransplantierte (46\%). Die am häufigsten berichteten Risikofaktoren für invasive Pilzinfektionen zu Beginn der Behandlung waren Einsatz eines zentralen Venenkatheters ( 37 von 41 Patienten, 90\%), Steroidgabe (37 von 41 Patienten, 90\%), kürzlich erfolgter Aufenthalt auf einer Intensivstation (36 von $41 \mathrm{~Pa}$ tienten, 88\%) und Dauer der Organtransplantation über $5 \mathrm{~h}$ (21 von 41 Patienten, 51\%). Bei 34 Patienten (83\%) lag eine systemische Kandidose und bei 10 Patienten (24\%) eine invasive Aspergillose vor. Am häufigsten betroffenen waren die Lungen (bei 21 von 41 Patienten, 51\%). Es erhielten 28 Patienten (68\%) Caspofungin als Monotherapie und 13 Patienten als Kombinationstherapie. Sechs Patienten (15\%) erhielten Caspofungin als Salvage-Therapie, in den meisten Fällen, weil sie auf die Vortherapie nicht angesprochen hatten. Während der Caspofungingabe erhielten 39 von 41 Patienten (95\%) auch Immunsuppressiva. Bei Patienten mit mindestens 5 Gaben Caspofungin (modifizierte „Intention-to-treat"-Population) betrug die Ge- samtansprechrate (partielles oder komplettes Ansprechen) am Ende der Caspofungintherapie $88 \%$ [ 29 von 33 Patienten; 95\%-Konfidenzintervall (95\%-KI) 72-97\%]. Hierbei sprachen $86 \%$ (19 von 22 Patienten) erfolgreich auf die Monotherapie mit Caspofungin und 10 von 11 Patienten (91\%) erfolgreich auf die Kombinationstherapie an. Es wurden weder unerwünschte Ereignisse noch Arzneimittelinteraktionen im möglichen, wahrscheinlichen oder gesicherten Zusammenhang mit der Caspofungintherapie berichtet. Die Gesamtüberlebensrate 7 Tage nach Beendigung der Behandlung mit Caspofungin betrug 79\% (26 von 33 Patienten; 95\%-KI 61-91\%).

Schlussfolgerung. Caspofungin war eine wirksame Behandlungsoption bei organtransplantierten Patienten mit wahrscheinlicher oder nachgewiesener invasiver Pilzerkrankung in Deutschland.

Schlüsselwörter

Pilzinfektion · Transplantation - Candida . Aspergillus · Caspofungin

\section{Caspofungin after solid organ transplantation in Germany. Observational study on treatment of invasive fungal infections}

\section{Abstract}

Background. This study was a pre-planned country-specific secondary analysis of results in Germany from a multinational multicenter observational study to retrospectively evaluate clinical outcomes with caspofungin in patients with probable and proven invasive fungal infection following solid organ transplantation (SOT).

Methods. Data were retrospectively collected on a single episode of invasive fungal infection (IFI) in patients who had a SOT between January 2004 and June 2007. Effectiveness was reported as the proportion of patients who received at least five doses of caspofungin with a favorable (complete or partial) response. Safety was assessed for patients who received at least one dose of caspofungin. Descriptive statistics were employed for all evaluations.

Results. A total of 41 SOT patients ( 27 male, 14 female; median age 56 years, median
APACHE II score at start of caspofungin therapy 23) were enrolled from 5 sites in Germany. Organs transplanted were mainly heart $(51 \%)$ and liver (46\%). Prevalent risk factors for IFI at baseline were use of central venous catheter ( 37 out of 41 patients, $90 \%$ ), steroid use (37 out of 41 patients, $90 \%$ ), recent stay in intensive care (36 out of 41 patients, $88 \%$ ), and duration of SOT procedure $>5$ hours ( 21 out of 41 patients, $51 \%$ ). Candidiasis was diagnosed in 34 patients ( $83 \%$ ) and aspergillosis in 10 patients $(24 \%)$. The lungs were the most common site of IFI ( 21 out of $41,51 \%$ ). Caspofungin as monotherapy was received by 28 patients ( $68 \%)$; 6 patients (15\%) received caspofungin as salvage therapy for IFI, in most cases because they were refractory to prior antifungal drugs. Immunosuppressants were administered with caspofungin in 39 out of 41 patients $(95 \%)$. In subjects with at least 5 doses of caspofungin (modified in- tention to treat population) the favorable response rate at the end of caspofungin therapy was $88 \%$ overall, 29 out of 33 patients; $95 \%$ confidence interval (95\%-Cl) 72-97\%), $86 \%$ (19 out of 22 patients) with monotherapy and $91 \%$ (10 out of 11 patients) with combination therapy. No (serious) adverse events or drug interactions related to treatment with caspofungin were reported. The overall survival rate was $79 \%$ ( 26 out of 33 patients; $95 \%-\mathrm{Cl} 61-91 \%$ ) at 7 days after completion of caspofungin treatment.

Conclusion. Caspofungin was found to be an effective treatment of probable and proven invasive fungal infections in patients following SOT in Germany.

\section{Keywords}

Fungal infection - Transplantation - Candida . Aspergillus · Caspofungin 


\begin{tabular}{|c|c|c|c|c|}
\hline & \multicolumn{4}{|l|}{ Population } \\
\hline & \multicolumn{2}{|c|}{$\begin{array}{l}\text { „All-patients-treated popula- } \\
\text { tion“ }\end{array}$} & \multicolumn{2}{|c|}{$\begin{array}{l}\text { „Modified all-patients-treated } \\
\text { population" }\end{array}$} \\
\hline & Anzahl (n) & Anteil (\%) & Anzahl (n) & Anteil (\%) \\
\hline Herz & 21 & 52 & 14 & 42 \\
\hline Leber & 18 & 44 & 17 & 52 \\
\hline Leber und Nieren & 1 & 2 & 1 & 3 \\
\hline Nieren und Pankreas & 1 & 2 & 1 & 3 \\
\hline Gesamt & 41 & 100 & 33 & 100 \\
\hline
\end{tabular}

- Daten zu Kombinationstherapie, zu möglichen Arzneimittelinteraktionen und zu unerwünschten Ereignissen im Zusammenhang mit der Caspofunginbehandlung.

Diese retrospektiv erhobenen Daten wurden nicht noch einmal durch Dritte evaluiert, und es wurden keine Resistenztestungen durchgeführt.

Ziel war die Beurteilung der Alltagswirksamkeit („effectiveness“), basierend auf einer Einschätzung des individuellen Ansprechens auf die Therapie durch den behandelnden Arzt am Ende der Caspofungingabe. Dazu musste der Studienpatient gemäß dem vordefinierten statistischen Analyseplan mindestens 5 Caspofungingaben erhalten haben („modified all-patients-treated population“). Die Ansprechrate auf die Therapie mit Caspofungin war die Hauptzielgröße der Untersuchung. Ein Ansprechen auf die Caspofungintherapie war als ein vollständiges oder teilweises Ansprechen definiert; ein Nichtansprechen der Therapie war als stabile Erkrankung, Therapieversagen bzw. Tod definiert. Grundlage hierfür war, dass jeder Patient gemäß der Einschätzung des behandelnden Arztes in eine der 4 etablierten Wirksamkeitskategorien für Registerstudien im Bereich invasive Pilzinfektionen $[14,18]$ eingeteilt wurde:

Ein vollständiges Ansprechen war hierbei als das am Ende der Caspofunginbehandlung durch den behandelnden Arzt festgestellte Verschwinden aller einer invasiven Pilzinfektion zuschreibbaren und vor Beginn der Therapie nachweisbaren klinischen sowie radiologischen Zeichen und Symptome definiert.

Teilweises Ansprechen bedeutete das am Ende der Caspofunginbehandlung durch den behandelnden Arzt festgestellte deutliche Zurückgehen der einer invasiven Pilzinfektion zuschreibbaren und vor Beginn der Therapie nachweisbaren klinischen sowie radiologischen Zeichen und Symptome.

Eine stabile Erkrankung bezog sich auf die am Ende der Caspofunginbehandlung durch den behandelnden Arzt festgestellte minimale oder ausbleibende Verringerung der einer invasiven Pilzinfektion zuschreibbaren und vor Beginn der Therapie nachweisbaren klinischen sowie radiologischen Zeichen und Symptome.

Als Therapieversagen wurde die am Ende der Caspofunginbehandlung durch den behandelnden Arzt festgestellte Verschlechterung der einer invasiven Pilzinfektion zuschreibbaren und vor Beginn der Therapie nachweisbaren klinischen sowie radiologischen Zeichen und Symptome oder ein Ableben des Patienten aufgrund invasiver Pilzerkrankung definiert.

Zusätzlich wurde der Vitalstatus bei Entlassung aus dem Krankenhaus erhoben.

In die Untersuchungen zur Sicherheit und Verträglichkeit wurden alle $\mathrm{Pa}$ tienten aufgenommen, die mindestens eine Caspofungingabe erhalten hatten (Allpatients-treated population). Die Erhebungen zur Sicherheit verlangten von allen Ärzten, jegliche nichtschwerwiegenden und schwerwiegenden klinischen bzw. labortechnischen unerwünschten Ereignisse, Arzneimittelinteraktionen, Therapieabbrüche oder Todesfälle zu berichten, die nach Einschätzung des behandelnden Arztes möglicherweise, wahrscheinlich oder definitiv mit der Caspofunginbehandlung zusammenhingen.
Die Patientenaufnahme erfolgte in zeitlich konsekutiver Folge. Die statistische Analyse wurde deskriptiv mit Berechnung binominal exakter 95\%-KI vorgenommen.

\section{Ergebnisse}

Insgesamt nahmen in den 5 deutschen Zentren 41 Patienten an der Studie teil. Es handelte sich um 14 Patientinnen (34\%) und 27 Patienten (66\%) mit vorwiegend Herz- oder Lebertransplantationen (- Tab. 2). Das Patientenalter zu Beginn der Caspofungintherapie lag im Median bei 56 Jahren (Mittelwert 54 Jahre, Minimum 25 Jahre bis Maximum 69 Jahre). Die Patienten wiesen zu Beginn der Beobachtung im Median 3 Begleiterkrankungen (Mittelwert 3,6, Minimum 1 bis Maximum 8) bzw. 8 Risikofaktoren (Mittelwert 7,9, Minimum 2 bis Maximum 18) auf. Die Dauer der Transplantationsoperation lag im Median bei $5 \mathrm{~h}$ (Mittelwert 5 h $17 \mathrm{~min}$, Minimum $2 \mathrm{~h} 6 \mathrm{~min}$ bis Maximum 13 h 12 min). Der Acute Physiology and Chronic Health Evaluation (APACHE) II Score der Patienten lag im Median bei 23 (Mittelwert 22,4, Minimum 14 bis Maximum 26).

Die Patienten wurden gemäß Fachinformation [19] initial im Median mit $70 \mathrm{mg}$ Caspofungin i.v. behandelt (Mittelwert 69,4 mg, Minimum 50 mg bis Maximum $100 \mathrm{mg}, \mathrm{n}=41$ ). Die Erhaltungsdosis (ab Tag 2) lag im Median bei $50 \mathrm{mg}$ (Mittelwert 49,1 mg, Minimum o mg bis Maximum $100 \mathrm{mg}, \mathrm{n}=41$ ). Die Dauer der Caspofunginbehandlung lag im Median bei 19 Tagen (Mittelwert 19,4 Tage, Minimum 7 Tage bis Maximum 57 Tage, $\mathrm{n}=33$ ). Hierbei waren die Monotherapiepatienten im Median 17 Tage mit Caspofungin (Mittelwert 18,7 Tage, Minimum 8 Tage bis Maximum 57 Tage, $n=22$ ) und die mit einer Kombination antimykotisch behandelten Patienten im Median $19 \mathrm{Ta}$ ge mit Caspofungin behandelt worden (Mittelwert 20,6 Tage, Minimum 7 Tage bis Maximum 37 Tage, $n=11$ ). Eine immunsuppressive Therapie erfolgte bei 39 der 41 Patienten (95\%; - Tab. 3), hauptsächlich mit Tacrolimus $(\mathrm{n}=25$, mittlere Dosis 7,44 mg, Minimum $1 \mathrm{mg}$ bis Maximum $25 \mathrm{mg}$ ). Kortikosteroide wurden bei 36 von 41 Patienten verabreicht, am 
häufigsten Prednisolon $(\mathrm{n}=18$, mittlere Dosis 85,5 mg, Minimum $7 \mathrm{mg}$ bis Maximum $750 \mathrm{mg}$ ) und Prednison ( $\mathrm{n}=8$, mittlere Dosis 110,25 mg, Minimum $7 \mathrm{mg}$ bis Maximum $750 \mathrm{mg}$ ).

Bei 20 Patienten lag nach Einschätzung des behandelnden Arztes eine wahrscheinliche Pilzinfektion und bei 21 Patienten eine nachgewiesenen Pilzinfektion vor (• Tab.4).

Unter den Patienten, die mindestens 5 Gaben Caspofungin erhalten hatten (Modified all-patients-treated population, n=33) lag die Ansprechrate nach Einschätzung des behandelnden Arztes am Ende der Caspofungintherapie bei 88\% (29 von 33 Patienten, 95\%-KI 72-97\%). Unter Caspofunginmonotherapie wurde ein Ansprechen bei 19 von 22 Patienten (86\%, 95\%-KI 65-97\%) erreicht. Unter der Kombination von Caspofungin mit einem anderen Antimykotikum wurde ein Ansprechen bei 10 von 11 Patienten (91\%, 95\%-KI 59-100\%) erzielt. Eine stabile Erkrankung wurde bei 3 von 33 Patienten (9\%) gesehen. Ein Patient wurde vom behandelnden Arzt als Therapieversager eingestuft: Hier handelte es sich um einen retransplantierten 64-jährigen Mann mit Lebertransplantation bei hepatozellulärem Karzinom. Im Respirationssekret war eine kombinierte Besiedlung mit Candida albicans, Aspergillus fumigatus und Aspergillus flavus festgestellt worden. Der Patient wurde 19 Tage mit einer Kombinationstherapie (Caspofungin und Voriconazol) behandelt, anschließend wurde die Kombinationstherapie beendet. Er verstarb 13 Tage nach Absetzen der Kombinationstherapie noch während des Krankenhausaufenthalts an Leberversagen; hierbei sahen die behandelnden Ärzte keinen ursächlichen Zusammenhang mit der antimykotischen Therapie. Die jeweiligen Ansprechraten, auch nach Grunderkrankungen und Risikofaktoren, die bei mindestens 10 Patienten auftraten, sind in - Tab. 4 aufgeführt.

Bezogen auf die Pilzinfektion sprachen insgesamt an: 4 von 5 Patienten (71\%) mit Aspergillus-Infektion; 2 von 3 Patienten mit Aspergillus- und Candida-Infektion (67\%) sowie 23 von 25 Patienten (92\%) mit Candida-Infektion (• Tab.5).

Die Gesamtüberlebensrate 7 Tage nach Beendigung der Behandlung mit Caspo-

\begin{tabular}{|c|c|c|c|c|c|c|c|c|}
\hline \multirow{4}{*}{ Immunosuppressive Therapie ${ }^{a}$} & \multicolumn{6}{|c|}{ Caspofungin } & \multirow{2}{*}{\multicolumn{2}{|c|}{ Gesamt }} \\
\hline & \multicolumn{4}{|c|}{ Monotherapie } & \multicolumn{2}{|c|}{$\begin{array}{l}\text { Kombinati- } \\
\text { onstherapie }\end{array}$} & & \\
\hline & \multicolumn{2}{|c|}{ Mit Steroid } & \multicolumn{2}{|c|}{ Ohne Steroid } & \multicolumn{2}{|c|}{ Mit Steroid } & \multirow[b]{2}{*}{$\begin{array}{l}\text { Anzahl } \\
\text { (n) }\end{array}$} & \multirow[b]{2}{*}{$\begin{array}{l}\text { Antei } \\
\text { (\%) }\end{array}$} \\
\hline & $\begin{array}{l}\text { Anzahl } \\
\text { (n) }\end{array}$ & $\begin{array}{l}\text { Anteil } \\
\text { (\%) }\end{array}$ & $\begin{array}{l}\text { Anzahl } \\
\text { (n) }\end{array}$ & $\begin{array}{l}\text { Anteil } \\
(\%)\end{array}$ & $\begin{array}{l}\text { Anzahl } \\
\text { (n) }\end{array}$ & $\begin{array}{l}\text { Anteil } \\
(\%)\end{array}$ & & \\
\hline Tacrolimus & 17 & 74 & 2 & 40 & 6 & 46 & 25 & 61 \\
\hline Ciclosporin A & 3 & 13 & 0 & 0 & 7 & 54 & 10 & 24 \\
\hline Mycophenolat-Mofetil & 2 & 9 & 0 & 0 & 1 & 8 & 3 & 7 \\
\hline Andere & 3 & 13 & 1 & 20 & 1 & 8 & 5 & 12 \\
\hline $\begin{array}{l}\text { Keine immunsuppressive } \\
\text { Therapie }\end{array}$ & 0 & 0 & 2 & 40 & 0 & 0 & 2 & 5 \\
\hline Gesamt & 23 & 100 & 5 & 100 & 13 & 100 & 41 & 100 \\
\hline
\end{tabular}

fungin lag bei 79\% (26 von 33 Patienten, 95\%-KI 61-91\%).

Bei keinem Patienten wurde die Caspofunginbehandlung wegen Arzneimittelinteraktionen zwischen Caspofungin und einem anderen Antimykotikum abgesetzt. Es fanden sich nach Einschätzung der behandelnden Ärzte keine unerwünschten Ereignisse, die möglicherweise, wahrscheinlich oder definitiv mit der Caspofunginbehandlung zusammenhingen. Die Gesamtdauer des Krankenhausaufenthalts (Gesamtbeobachtungszeitraum) bei diesen Patienten betrug im Median 59 Tage (Mittelwert 83,5 Tage, Minimum 9 Tage bis Maximum 398 Tage, $\mathrm{n}=33$ ); hiervon lag der Median bei den monotherapierten Patienten bei 59 Tagen ( $\mathrm{n}=\mathbf{2 2}$, Mittelwert 87,4 Tage, Minimum 9 Tage bis Maximum 398 Tage), bei den kombiniert behandelten Patienten bei 56 Tagen (Mittelwert 75,7 Tage, Minimum 22 Tage bis Maximum 228 Tage). Insgesamt verstarben 12 von 33 Patienten (36\%) innerhalb des Beobachtungszeitraums - in keinem dieser Fälle wurde vonseiten der behandelnden Ärzte ein Zusammenhang mit der Caspofungintherapie berichtet. Bei einem Patienten war nach Ansicht des behandelnden Arztes die Todesursache eine Pilzinfektion. Weiterhin wurden als Todesursachen genannt: Multiorganversagen (5 Patienten), Multiorganversagen wegen einer bakteriellen Sepsis auf dem Boden einer Transplantatabstoßung (1 Patient), Bakteriämie mit septischem Schock (1 Patient), Infektion mit Enterococcus fae- cium und Methillicin-resistentem Staphylococcus aureus (MRSA, 1 Patient), diffuse Hämorrhagie (1 Patient), Graft versus Host Disease (GvHD, 1 Patient) sowie Leberversagen (1 Patient). Alle anderen 21 von 33 Patienten (64\%) wurden von dem Studienzentrum innerhalb der Beobachtungszeit entlassen; hierbei konnten 9 von 33 Patienten $(27 \%)$ in eine andere Institution und 12 von 33 Patienten (36\%) nach Hause entlassen werden.

\section{Diskussion}

Caspofungin zeigte in dieser retrospektiven Beobachtungsstudie mit einer Gesamtansprechrate von $88 \%$ nach Einschätzung der behandelnden Ärzte eine gute Alltagswirksamkeit (Effectiveness) bei der Therapie von Patienten nach SOT mit wahrscheinlicher oder bewiesener Infektionen durch Candida- oder AspergillusSpezies (Modified all-patients-treated population). Auch bei der All-patients-treated population lag die Ansprechrate mit $83 \%$ sehr hoch (34 von 41 Patienten, Posthoc-Analyse). In der hier untersuchten Population schwer erkrankter Patienten mit immunsuppressiver Therapie wurde von den behandelnden Ärzten von keinen unerwünschten Ereignissen oder Arzneimittelinteraktion in möglichem, wahrscheinlichem oder gesichertem Zusammenhang mit der Therapie mit Caspofungin berichtet.

Bei Caspofungin handelt es sich um den ersten zugelassenen Wirkstoff aus 
Tab. 4 Alltagswirksamkeit nach Einschätzung des behandelnden Arztes am Ende der Caspofungintherapie bei 33 organtransplantierten Patienten mit wahrscheinlicher oder gesicherter invasiver Pilzerkrankung nach Erreger („,modified all-patients-treated population“)

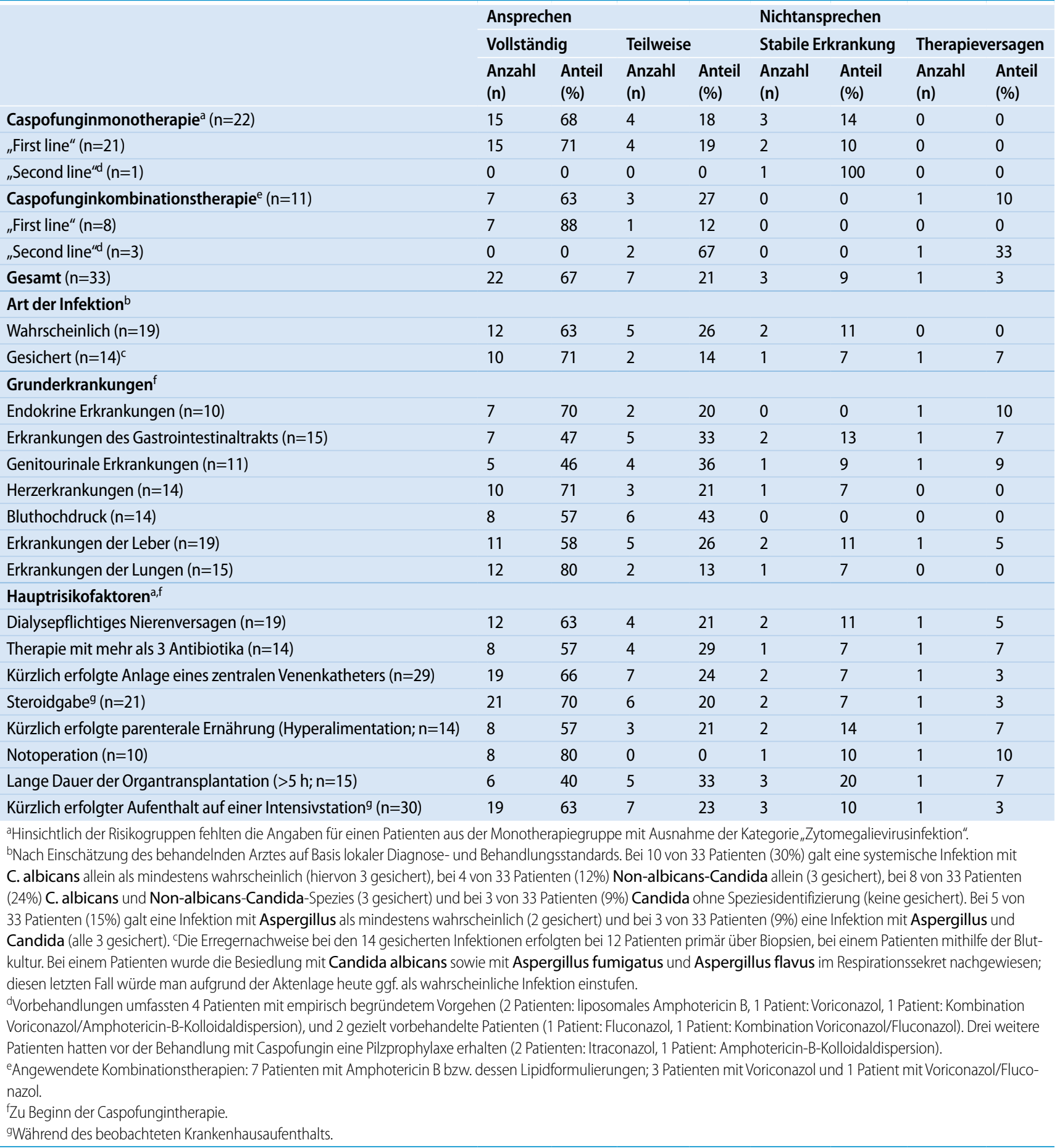

der Gruppe der Echinocandine. Caspofungin wirkt antimykotisch durch Inhibition des zellmembranständigen Enzyms $\beta$-(1,3)-D-Glukan-Synthase, das ein essenzielles Enzym für die Zellwandsynthese bei vielen Pilzen ist, z. B. bei Candida- und Aspergillus-Spezies. Caspofungin ist in Europa bei erwachsenen und pädiatrischen Patienten zur Behandlung der invasiven Kandidose, zur empirischen Therapie bei Verdacht auf Infektionen durch Pilze (wie Candida oder Aspergillus) bei neutropenen Patienten mit Fieber und zur Behandlung der invasiven Aspergillose bei Patienten, die auf andere Antimykotika (Amphoterin B, Itraco- nazol) nicht ansprechen oder diese nicht vertragen, zugelassen [17].

Insgesamt liegen nur wenige Daten zur antimykotischen Behandlung von Patienten nach SOT vor. Aussagefähige Daten aus größeren Studien existieren nur für die antimykotische Prohylaxe nach Lungen- und Lebertransplantation [3, 21]. 
In der relevanten Zulassungsstudie waren 6 der 109 mit Caspofungin behandelten Patienten Transplantationspatienten (5 nierentransplantierte Patienten, 1 lebertransplantierter Patient), die alle primär mit Caspofungin behandelt worden waren. Die Therapie war in 5 der 6 Fälle erfolgreich [20].

Aus den übrigen vorliegenden randomisierten Studien wurden zusätzlich die teilnehmenden Intensivpatienten herausgelöst betrachtet $(n=97,81 \%$ davon zur Therapie einer Kandidämie; [5]). Dabei zeigten $68 \%$ der auf der Intensivstation behandelten Patienten unter Caspofungingabe ein positives Ansprechen. Intensivpatienten hatten insgesamt ein höheres Risiko zu versterben als Patienten auf peripheren Stationen. Für diese Intensivpatienten lag die Gesamtletalität bei 45\% für Caspofungin, während die der CandidaInfektion zuschreibbare Letalität in der Caspofungingruppe bei $5 \%$ lag. Caspofungin zeigte auch bei selteneren Formen einer invasiven Kandidose in $81 \%$ der Fälle (z. B. Peritonitis oder Endokarditis, ohne Nachweis einer Kandidämie, $n=48$ ) ein positives Ansprechen am Ende der Therapie. Dabei war Caspofungin auch in höheren Dosierungen von bis zu $100 \mathrm{mg} /$ Tag gut verträglich [2]. Die hier vorgelegten multizentrischen deskriptiven Daten von Transplantationspatienten mit invasiver Pilzerkrankung zeigen in eine vergleichbare Richtung.

Bemerkenswert an den Studienergebnissen ist die relativ hohe Rate von hier berichteten Fällen einer invasiven Aspergillose. Bei etwa einem Drittel der in dieser Studie mit Caspofungin behandelten Fälle lag eine wahrscheinliche oder gesicherte Infektion durch Aspergillus-Spezies vor, teilweise kombiniert mit einer Candida-Infektion. Nach Einschätzung des behandelnden Arztes sprachen 6 von 8 Patienten $(75 \%)$ positiv an. Die Wirksamkeit gegen Aspergillus-Spezies bewies Caspofungin erstmals in einer Studie zur Therapie einer bewiesenen oder wahrscheinlichen Aspergillose bei Patienten $(n=83)$, die ein Therapieversagen $(86 \%$ der Fälle) oder Unverträglichkeit gegenüber Amphotericin B oder Azolen zeigten. Hier war Caspofungin in $45 \%$ der Fälle wirksam (vollständige Remission: 5\%; [15]). Bei dem überwiegenden Anteil

\begin{tabular}{|c|c|c|c|c|c|}
\hline & $\begin{array}{l}\text { First-line- } \\
\text { Therapie }\end{array}$ & $\begin{array}{l}\text { Second-line- } \\
\text { Therapie }\end{array}$ & $\begin{array}{l}\text { Mono- } \\
\text { therapie }\end{array}$ & $\begin{array}{l}\text { Kombinati- } \\
\text { onstherapie }\end{array}$ & Gesamt \\
\hline Aspergillus & 4 von 4 & 0 von 1 & 3 von 4 & 1 von 1 & 4 von 5 \\
\hline Aspergillus und Candida & 2 von 2 & 0 von 1 & - & 2 von 3 & 2 von 3 \\
\hline Candida albicans & 8 von 9 & 0 von 1 & 5 von 7 & 3 von 3 & 8 von 10 \\
\hline Non-albicans-Candida-Spezies ${ }^{\mathrm{a}}$ & 10 von 10 & 2 von 2 & 10 von 10 & 2 von 2 & 12 von 12 \\
\hline $\begin{array}{l}\text { Candida-Spezies ohne Spezies- } \\
\text { identifizierung }\end{array}$ & 3 von 3 & - & 1 von 1 & 2 von 2 & 3 von 3 \\
\hline Gesamt & 27 von 28 & 2 von 5 & 19 von 22 & 10 von 11 & 29 von 33 \\
\hline
\end{tabular}

der Patienten lag eine hämatoonkologische Erkrankung zugrunde. Nur $11 \%$ der Patienten (9 von 83 Patienten) hatten sich im Vorfeld einer SOT unterzogen; bei 4 dieser Fälle war ein positives Ansprechen auf die sekundäre Caspofungintherapie $\mathrm{zu}$ verzeichnen. Insgesamt wurden 16 transplantierte Patienten in verschiedene Phase-II/III-Studien zur sekundären Therapie der invasiven Aspergillose aufgenommen [20]. In der Hälfte der Fälle fand sich ein positives Ansprechen. In einer kleinen Fallserie von 12 Patienten nach Herz- bzw. Lungentransplantation mit pulmonaler Aspergillose konnte Caspofungin in 10 Fällen erfolgreich eingesetzt werden [8]. Über unerwünschte labortechnische Ereignisse einschließlich Leberwerte in möglichem, wahrscheinlichem oder gesichertem Zusammenhang mit der Caspofungingabe wurde von den behandelnden Ärzten bei dem hier vorgestellten Patientengut nicht berichtet.

Kombinationstherapien erscheinen aufgrund der hohen Letalitätsrate bei der Behandlung der invasiven Aspergillose insbesondere bei Transplantationspatienten attraktiv. Bisher untersuchten nur wenige, nichtrandomisierte Studien den tatsächlichen klinischen Nutzen einer Kombination von verschiedenen Antimykoti$\mathrm{ka}$ in dieser Population [11, 12, 13, 22, 27]. Diese Studie bestätigt, dass in Deutschland nach einer Transplantation nicht selten eine antimykotische Kombinationstherapie verordnet wird. Trotz numerisch vergleichbarer Ansprechraten ist die vorgestellte Untersuchung allerdings nicht geeignet, auf die relative Wirksamkeit der Monotherapie gegenüber der Kombina- tionstherapie mit Caspofungin zu schließen.

Obwohl insgesamt nur eine relativ geringe Zahl von 41 Patienten betrachtet wurde, handelt es sich um das größte bisher aus Deutschland berichtete multizentrische Kollektiv organtransplantierter Patienten mit behandelter invasiver Pilzerkrankung. Die Ergebnisse dieser Arbeit zeigen, dass Caspofungin auch in der Gruppe organtransplantierter Patienten eine mit den bisher veröffentlichten Studien vergleichbare Alltagswirksamkeit besitzt. Zusätzlich erweist sich Caspofungin bei den hier untersuchten Patienten als gut verträglich; dies ist für die Gruppe dieser schwer erkrankten Personen (Median des APACHE-II-Scores: 23; 4 Patienten erhielten keine immunsuppressive Therapie mehr und 2 kein Kortikoid), bei denen zudem regelhaft Organinsuffizienzen vorliegen, von großer Bedeutung. Auf für Caspofungin beschriebene Interaktionen [4] fanden sich bei dem hier untersuchten Kollektiv keine Hinweise. Insbesondere berichtete keiner der behandelnden Ärzte bei diesen mit Tacrolimus und anderen Immunsuppressiva behandelten $\mathrm{Pa}$ tienten von Interaktionen und/oder unerwünschten Ereignissen mit einem möglichen, wahrscheinlichen oder definitiven Zusammenhang mit der Caspofunginbehandlung.

Als Beobachtungsstudie bezieht sich diese Arbeit in der Beurteilung der Alltagswirksamkeit auf die alleinige Einschätzung des behandelnden Arztes. Obwohl insgesamt eine relativ geringe Zahl von 41 Patienten betrachtet wurde, gibt diese Studie einen klaren Hinweis auf die 
Wirksamkeit von Caspofungin in der Behandlung von Pilzinfektionen in dieser noch kaum untersuchten Population organtransplantierter Patienten.

\section{Fazit für die Praxis}

\section{Caspofungin war eine wirksame und gut verträgliche Option für die Behandlung wahrscheinlicher oder nachgewiesener invasiver Pilzerkrankungen durch Can- dida oder Aspergillus bei Patienten nach SOT in Deutschland.}

\section{Korrespondenzadresse \\ Dr. C. Lichtenstern}

Klinik für Anästhesiologie und Operative Intensivmedizin, Universitätsklinikum Gießen und Marburg, Standort Gießen Rudolf-Buchheim Straße 7, 35385 Gießen christoph.lichtenstern@chiru.med.unigiessen.de

Open Access. This article is distributed under the terms of the Creative Commons Attribution Noncommercial License which permits any noncommercial use, distribution, and reproduction in any medium, provided the original author(s) and source are credited.

Interessenkonflikt. Finanzierung der Studie: Merck \& Co., Inc, Whitehouse Station, NJ, USA. C.L. gibt Vorträge für MSD Sharp \& Dohme GmbH sowie Astra-Zeneca und erhielt Reisekostenunterstützung von Essex Pharma, Pfizer und. Wyeth. J.P. gibt Vorträge für Astellas, Genzyme, MSD Sharp \& Dohme GmbH und Novartis. U.S. erhielt Forschungsunterstützung von Actelion, Fresenius, MSD Sharp \& Dohme $\mathrm{GmbH}$, Miltenyi, und Novartis sowie Reisekostenunterstützung von Actelion, Astellas, Bayer, Fresenius, MSD Sharp \& Dohme GmbH, Miltenyi, Novartis und Pfizer. M.S. erhielt Reisekostenunterstützung von MSD Sharp \& Dohme GmbH. W.K. gibt Vorträge für MSD Sharp \& Dohme GmbH, Novartis, Astellas, Pfizer sowie Bayer und erhielt Reisekostenunterstützung. P.K. und K.J.K. sind Angestellte der Firma MSD Sharp \& Dohme $\mathrm{GmbH}$, der deutschen Tochtergesellschaft von Merck \& Co., Inc., dem Hersteller von Caspofungin. M.A.W. hält Vorträge für Astellas, Astra-Zeneca, Bayer, Braun, Essex Pharma, Glaxo, Gilead, Janssen-Cilag, Lilly, MSD Sharp \& Dohme GmbH, Novartis, Pfizer, Wyeth und ZLB Behring. Er ist beratend tätig für Essex Pharma, MSD Sharp \& Dohme GmbH, Pfizer und Wyeth. M.W. erhielt Forschungsunterstützung von Bayer und Novartis, ist beratend tätig für Astellas, MSD Sharp \& Dohme GmbH und Bayer und gibt Vorträge für Bayer, MSD Sharp \& Dohme GmbH und Novartis.

\section{Literatur}

1. Ascioglu S, Rex JH, De Pauw B et al (2002) Defining opportunistic invasive fungal infections in immunocompromised patients with cancer and hematopoietic stem cell transplants: an international consensus. Clin Infect Dis 34:7-14
2. Cornely OA, Lasso M, Betts R et al (2007) Caspofungin for the treatment of less common forms of invasive candidiasis. J Antimicrob Chemother 60:363-369

3. Cruciani M, De Lalla F, Mengoli C (2005) Prophylaxis of Candida infections in adult trauma and surgical intensive care patients: a systematic review and meta-analysis. Intensive Care Med 31:1479-1487

4. Denning DW (2003) Echinocandin antifungal drugs. Lancet 362:1142-1151

5. Dinubile MJ, Lupinacci RJ, Strohmaier KM et al (2007) Invasive candidiasis treated in the intensive care unit: observations from a randomized clinical trial. J Crit Care 22:237-244

6. Fortun J, Martin-Davila P, Moreno S et al (2002) Risk factors for invasive aspergillosis in liver transplant recipients. Liver Transpl 8:1065-1070

7. Gavalda J, Len O, San JR et al (2005) Risk factors for invasive aspergillosis in solid-organ transplant recipients: a case-control study. Clin Infect Dis 41:52-59

8. Groetzner J, Kaczmarek I, Wittwer T et al (2008) Caspofungin as first-line therapy for the treatment of invasive aspergillosis after thoracic organ transplantation. J Heart Lung Transplant 27:1-6

9. Hagerty JA, Ortiz J, Reich D et al (2003) Fungal infections in solid organ transplant patients. Surg Infect (Larchmt) 4:263-271

10. Husain S, Tollemar J, Dominguez EA et al (2003) Changes in the spectrum and risk factors for invasive candidiasis in liver transplant recipients: prospective, multicenter, case-controlled study. Transplantation 75:2023-2029

11. Kontoyiannis DP, Boktour M, Hanna H et al (2005) Itraconazole added to a lipid formulation of amphotericin B does not improve outcome of primary treatment of invasive aspergillosis. Cancer 103:2334-2337

12. Kontoyiannis DP, Hachem R, Lewis RE et al (2003) Efficacy and toxicity of caspofungin in combination with liposomal amphotericin B as primary or salvage treatment of invasive aspergillosis in patients with hematologic malignancies. Cancer 98:292-299

13. Kontoyiannis DP, Ratanatharathorn V, Young JA et al (2009) Micafungin alone or in combination with other systemic antifungal therapies in hematopoietic stem cell transplant recipients with invasive aspergillosis. Transpl Infect Dis 11:89-93

14. Linden P, Williams P, Chan KM (2000) Efficacy and safety of amphotericin $\mathrm{B}$ lipid complex injection $(A B L C)$ in solid-organ transplant recipients with invasive fungal infections. Clin Transplant 14:329_ 339

15. Maertens J, Raad I, Petrikkos G et al (2004) Efficacy and safety of caspofungin for treatment of invasive aspergillosis in patients refractory to or intolerant of conventional antifungal therapy. Clin Infect Dis 39:1563-1571

16. Marik PE (2006) Fungal infections in solid organ transplantation. Expert Opin Pharmacother 7:297305

17. MSD, Sharp \& Dohme GmbH (Haar, Juli 2009) Fachinformation CANCIDAS ${ }^{\circledR} 50 \mathrm{mg} / 70 \mathrm{mg}$ Pulver für ein Konzentrat zur Herstellung einer Infusionslösung

18. Pappas PG (2005) Amphotericin B lipid complex in the treatment of invasive fungal infections: results of the Collaborative Exchange of Antifungal Research (CLEAR), an industry-supported patient registry. Clin Infect Dis 40 [Suppl 6]:379-383
19. Patel R, Portela D, Badley AD et al (1996) Risk factors of invasive Candida and non-Candida fungal infections after liver transplantation. Transplantation 62:926-934

20. Petrovic J, Ngai A, Bradshaw S et al (2007) Efficacy and safety of caspofungin in solid organ transplant recipients. Transplant Proc 39:3117-3120

21. Playford EG, Webster AC, Sorell TC et al (2004) Antifungal agents for preventing fungal infections in solid organ transplant recipients. Cochrane Database Syst Rev:CD004291

22. Popp Al, White MH, Quadri T et al (1999) Amphotericin B with and without itraconazole for invasive aspergillosis: A three-year retrospective study. Int J Infect Dis 3:157-160

23. Salavert M (2008) Prophylaxis, pre-emptive or empirical antifungal therapy: which is best in nonlung transplant recipients? Int J Antimicrob Agents 32 [Suppl 2]:149-153

24. Silveira FP, Husain S (2007) Fungal infections in solid organ transplantation. Med Mycol 45:305-320

25. Singh N (2004) Antifungal prophylaxis in solidorgan transplant recipients: considerations for clinical trial design. Clin Infect Dis 39 [Suppl 4]:200 206

26. Singh N, Avery RK, Munoz P et al (2003) Trends in risk profiles for and mortality associated with invasive aspergillosis among liver transplant recipients. Clin Infect Dis 36:46-52

27. Singh N, Limaye AP, Forrest G et al (2006) Combination of voriconazole and caspofungin as primary therapy for invasive aspergillosis in solid organ transplant recipients: a prospective, multicenter, observational study. Transplantation 81:320-326

28. Singh N, Wagener MM, Marino IR et al (2002) Trends in invasive fungal infections in liver transplant recipients: correlation with evolution in transplantation practices. Transplantation 73:63-67

29. Winkler M, Pratschke J, Schulz U et al (2010) Caspofungin for post solid organ transplant invasive fungal disease: results of a retrospective observational study. Transpl Infect Dis 12:230-237 